\title{
Chest CT study of fifteen COVID-19 patients with positive RT-PCR retest results after discharge
}

\author{
Chenxi $\mathrm{Li}^{1}$, Fan $\mathrm{Luo}^{1}$, Liqiu Xie ${ }^{1}$, Yueqin $\mathrm{Gao}^{1}, \mathrm{Na}$ Zhang ${ }^{1}$, Bing $\mathrm{Wu}^{2}$ \\ ${ }^{1}$ Department of Radiology, Public Health Clinical Center of Chengdu, Chengdu, 610000, China; ${ }^{2}$ Department of Radiology, China West Hospital, \\ Sichuan University, Chengdu 610041, China
}

Correspondence to: Na Zhang. Department of Radiology, Public Health Clinical Center of Chengdu, Jingming Road No. 377 , Jinjiang District, Chengdu 610000, China. Email: 147841845@qq.com.

\begin{abstract}
Background: Our hospital is a designated institution for COVID-19 patients in Chengdu, China. This study aimed to analyze the clinical and chest CT features of 15 COVID-19 patients with positive reverse transcription-polymerase chain reaction (RT-PCR) retest results after discharge. Patients who met the current standards of discharge could still carry the SARS-CoV-2 virus.

Methods: Clinical manifestations, laboratory data, and chest CT images were retrospectively reviewed and analyzed.

Results: The most common symptoms at Covid-19COVID-19 initial onset were fever (12/15, 80\%) and cough $(11 / 15,73.3 \%)$. Most of the patients had a normal white blood cells $(12 / 15,80 \%)$, neutrophils (12/15, 80\%), and lymphocytes count (13/15, 86.7\%); some patients had increased C-reactive protein (CRP) $(5 / 15,33.3 \%)$, and increased lactate dehydrogenase (LDH) $(4 / 15,26.7 \%)$ during first admission. Five patients $(33.3 \%)$ had a cough before their first discharge. The average interval from the first discharge to readmission was 17 days (range, 9-30 days). At re-admission, two (13.3\%) patients presented with cough, and one $(6.6 \%)$ had chest pain with anxiety. At re-admission, all patients had normal clinical results except five (33.3\%) patients had increased CRP compared with first discharging, two (13.3\%) patients had increased neutrophils count, and one (6.6\%) had increased CRP. The majority of patients had normal procalcitonin. Ground glass opacities (GGOs) and reticulation in the peripheral and subpleural areas were the most common CT manifestations, and six patients (40\%) showed a transformation from reticulation to GGOs when re-admitted.
\end{abstract}

Conclusions: There may be no specific clinical characteristics to predict the re-detectability of the virus. A regular medical observation and a bi-monthly follow-up is recommended.

Keywords: COVID-19; reverse transcription-polymerase chain reaction (RT-PCR); chest computed tomography (chest CT)

Submitted Apr 04, 2020. Accepted for publication May 11, 2020.

doi: 10.21037/qims-20-530

View this article at: http://dx.doi.org/10.21037/qims-20-530

\section{Introduction}

The severe acute respiratory syndrome coronavirus 2 (SARS-CoV-2) was first discovered in Wuhan, China, in late December 2019. It spreads quickly and has infected more than 80,000 people in the whole country. All discharged patients are required to stay in quarantine for at least 2 weeks (1). Our hospital is a designated hospital in
Chengdu, Sichuan, China, for patients with COVID-19. We hospitalized and treated 105 patients during January 17 to March 20, 2020. All patients discharged must have meet the criteria according to the latest version of the diagnostic and treatment criteria (2): (I) body temperature should remain normal for more than 3 days; (II) obvious alleviation of respiratory symptoms; (III) remarkable absorption of 
infection signs on chest CT images; (IV) two consecutive (after a 24-hour interval) negative reverse transcriptionpolymerase chain reaction (RT-PCR) tests. A total of 15 discharged patients returned to our center because of their follow-up RT-PCR tests showed positive results again.

Little is known about the follow-up of COVID-19. If patients who are discharged and released from isolation retest positive, the risk of human-to-human transmission would be difficult to avoid (3). Therefore, it is very important to pay attention to this kind of disease management. We analyzed these 15 cases to determine their relative CT image features and their correlation with RT-PCR results, hoping to provide ideas and insights for follow-up control and management. All these patients received chest CT exams throughout their clinical course, and their clinical and laboratory results were evaluated.

\section{Methods}

A total of 105 patients with confirmed COVID-19 were admitted to our hospital in the past 2 months until the date of study. They had positive RT-PCR assay results for SARS-CoV-2 nucleic acids. All patients discharged met the criteria according to the latest version of the diagnostic and treatment criteria (2): (I) body temperature should remain normal for more than 3 days; (II) obvious alleviation of respiratory symptoms; (III) remarkable absorption of infection signs on chest CT images; (IV) two consecutive (after a 24-hour interval) negative RT-PCR tests.

As regular follow-up, the patients were asked to return for PCR tests every 2 weeks. Up to now, 85 patients returned to our hospital for follow-up PCR test as instructed (patients who went to other hospitals were not followed-up on). 15 patients $(15 / 85,17.6 \%)$; who were discharged after meeting the current standards, were readmitted because of positive RT-PCR retest results.

All 15 patients underwent chest CT tests during their course of hospitalization, especially on the day before their first discharge and on the day of re-admission. The patients' chest images were obtained using the multi-slice spiral CT scanner (Somatom go.Top; Siemens Healthineers) in our department. A scanning protocol for the highresolution computed tomography (HRCT) scan was set with a tube voltage of 100 to $120 \mathrm{kV}$ and automatic tube current modulation. The reconstruction condition had a slice thickness of $1.0 \mathrm{~mm}$, and an interval of $1.0 \mathrm{~mm}$. The coverage area was taken from the lung apex to the diaphragm in the axial plane after the patients inspired and held their breath.

We compared the CT images on the day before their first discharge (RT-PCR confirmed negative) with images on the day of re-admission (RT-PCR confirmed positive). Two radiologists; each with 10 and 20 years of medical and radiological diagnostic experience, respectively reviewed and recorded the HRCT images independently using a picture archiving and communication system (PACS). Discrepancies were resolved by consensus. The display settings for the reviews were as follows: lung (window width, 1,500 HU; window level, -500 HU); mediastinal (window width, $350 \mathrm{HU}$; window level, $50 \mathrm{HU}$ ). For all 15 patients, chest CT images were evaluated for: (I) ground-glass opacities (GGOs), consolidation, reticulation, subpleural lines, and small nodules; (II) the location of the lesions (peripheral, non-peripheral, posterior, non-posterior); (III) other manifestations: pleural effusion or lymphopathy; and (IV) the changes in the lung lesions, which were described according to the specific manifestations.

The patients' clinical manifestations and laboratory results upon first admission, before first discharge, and upon re-admission were collected and analyzed retrospectively.

None of the included patients had lung surgeries, infections, tumors, or other lung diseases.

\section{Results}

A total of 15 patients, four men $(26.7 \%)$ and 11 women $(73.3 \%)$ with an average age of 48 years old (range, $23-$ 68 years) were included. Six patients $(40 \%)$ had been to Wuhan, 6 patients $(40 \%)$ had close contact with people from Wuhan, and the rest $(3 / 15,20 \%)$ had no clear contact history. The most common symptoms were fever (12/15, $80 \%)$, cough $(11 / 15,73.3 \%)$, and other symptoms including dyspnea $(2 / 15,13.3 \%)$, headache $(2 / 15,13.3 \%)$, chest pain $(2 / 15,13.3 \%)$, chills $(2 / 15,13.3 \%)$, and digestive symptoms $(1 / 15,6.7 \%)$. Most of the patients had a normal white blood cells count, neutrophils count and lymphocytes count throughout their whole course. Some patients had increased C-reactive protein (CRP) $(5 / 15,33.3 \% ; 5 / 15,33.3 \% ; 1 / 15$, $6.7 \%$; respectively). Four patients $(26.7 \%)$ had increased lactate dehydrogenase (LDH) and 1 patient $(6.7 \%)$ had lower procalcitonin at initial onset, while all patients had normal LDH and procalcitonin results in later tests.

Before their first discharge, only 5 patients (33.3\%) still had cough, while others had no symptoms.

The average interval from their first discharge to readmission was 17 days $( \pm 6.72$, ranging from $9-30$ days $)$. 
Table 1 Demographic information, clinical manifestation, and laboratory data of 15 patients at first admissions first discharge and second admissions

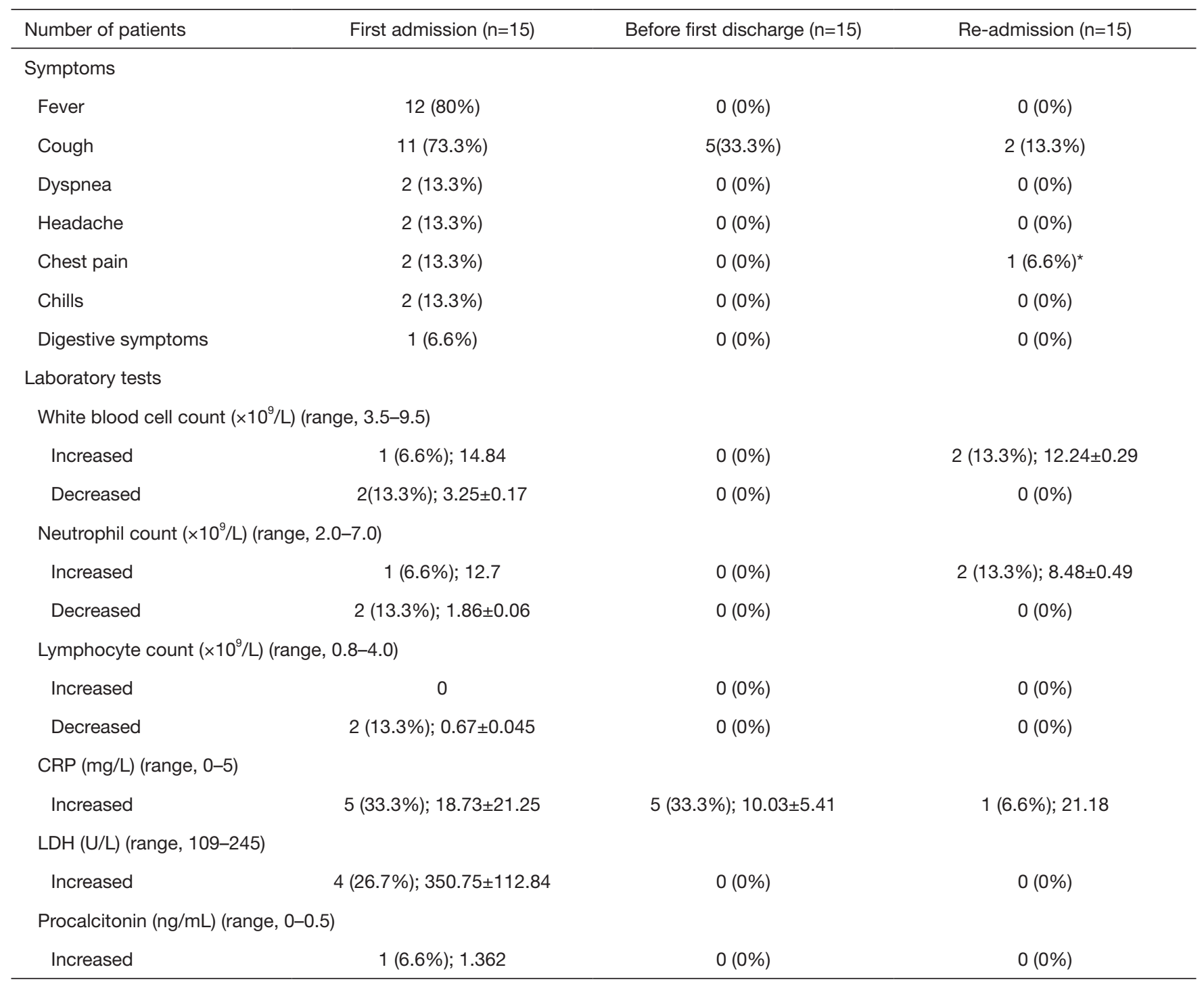

*, this patient complained of chest pain had anxiety. CRP, C-reactive protein; LDH, lactate dehydrogenase.

However, two patients $(13.3 \%)$ had cough, one $(6.6 \%)$ had dyspnea, and one $(6.6 \%)$ had chest pain during readmission.

Their demographic information, clinical manifestations, and laboratory data are tabulated in Table 1. Table 2 shows the main chest CT scans at admission and readmission, along with their respective findings. The lung segments affected reduced from an average of seven to an average of five segments affected at re-admittance. The peripheral location was affected most frequently $(13 / 15,86.7 \% ; 12 / 15$, $80 \%)$. Lesions were also more common in the posterior area $(10 / 15,66.7 \% ; 9 / 15,60 \%)$. GGOs were the most common manifestations $(14 / 15,93.3 \% ; 12 / 15,80 \%$, respectively), followed by reticulation $(10 / 15,66.7 \% ; 4 / 15$, $26.7 \%$, respectively). A few patients had pleural lines (4/15, $26.7 \% ; 2 / 15,13.3 \%)$ and small nodules $(2 / 15,13.3 \%, 2 / 15$, $13.3 \%)$. Only one patient $(6.7 \%)$ had consolidation, and none of the patients showed pleural effusion or lymphopathy. Eight patients (53.3\%) exhibited decreased lesions and different degrees of absorption, and one patient $(6.7 \%)$ had no changes. Two patients (13.3\%) had negative findings on their chest CT even when their retest RT-PCR results were 
Table 2 Main CT findings of the 15 patients

\begin{tabular}{lcc}
\hline Characteristics & First discharge, $\mathrm{n}(\%)$ & Re-admission, $\mathrm{n}(\%)$ \\
\hline Number of segments involved & $7(46.7)$ & $5(33.3)$ \\
Location & & $12(80.0)$ \\
Peripheral & $13(86.7)$ & $2(13.3)$ \\
Non-peripheral & $2(13.3)$ & $9(60.0)$ \\
Posterior & $10(66.7)$ & $5(33.3)$ \\
Non-posterior & $5(33.3)$ & $12(80.0)$ \\
GGO & $14(93.3)$ & $0(0.0)$ \\
Consolidation & $1(6.7)$ & $4(26.7)$ \\
Reticulation & $10(66.7)$ & $2(13.3)$ \\
Pleural lines & $4(26.7)$ & $2(13.3)$ \\
Small nodule & $2(13.3)$ & $0(0.0)$ \\
Pleural effusion & $0(0.0)$ & $0(0.0)$ \\
Lymphopathy & $0(0.0)$ & 0.0 \\
\hline
\end{tabular}

positive. In 6 patients (40\%), their reticulations changed to GGOs at re-admission, but all of them showed improvements after their second hospitalization (Figures 1,2).

\section{Discussion}

SARS-CoV-2 is the third largest coronavirus outbreak, after severe acute respiratory syndrome (SARS) and Middle East respiratory syndrome (MERS). SARS and MERS also showed human-to-human transmission, but there were no large outbreaks reported in other areas beyond the initial epidemic (4), which implied that COVID-19 is more contagious and riskier for humans. We still do not know enough about this virus, and there is still a lack of knowledge in terms of follow-up management. In our study, we reported and analyzed the clinical and radiological findings from 15 COVID-19 patients with positive RTPCR retest results upon follow-up, which have not been described systematically in previous literature.

The most common initial clinical manifestations seen in these 15 patients were fever and cough, which was consistent with earlier research (5). Majority of the patients had a normal white blood cells count, a few patients had increased CRP and LDH, and only one patient had abnormal procalcitonin when first admitted, which also was consistent with a previous study (4). Patients generally had few symptoms before their first discharge, and most of them did not have symptoms during re-admission. Two patients (13.3\%) had higher white blood cells and neutrophils counts than at their first discharge. Ye et al. reviewed 55 patients confirmed with COVID-19 and found that 5 (9\%) patients who were discharged retested positive again for the virus using RT-PCR. Their study concluded that there may not be any specific clinical characteristics that can predict the recurrence of the virus (6); however, this study did not mention the radiological features or changes in these patients. A regular medical surveillance study was conducted among 62 recovered medical staffs with COVID-19 (7), and two positive cases $(3.23 \%)$ had been discovered. The study hypothesizes that it would take some time for the human immune system to eliminate the virus completely, and if a patient's immunity decreases, there is a risk of relapse. In our study, the 15 patients' physical conditions were stable with no obvious decreased immunity, and there was no deterioration seen in the CT images; therefore, it is difficult to relate the outcome to the immune system. Xie et al. suggested that low viral loads, different methods of collecting specimens, and laboratory errors could lead to false-negative RT-PCR tests (8). The bronchoalveolar lavage fluid (BALF) specimen test might be the most accurate method, but the exposure risk is relatively high (9); therefore, oropharyngeal or nasopharyngeal swab tests are adopted more often in clinical settings. However, these sample types could lead to a high rate of false-negative 

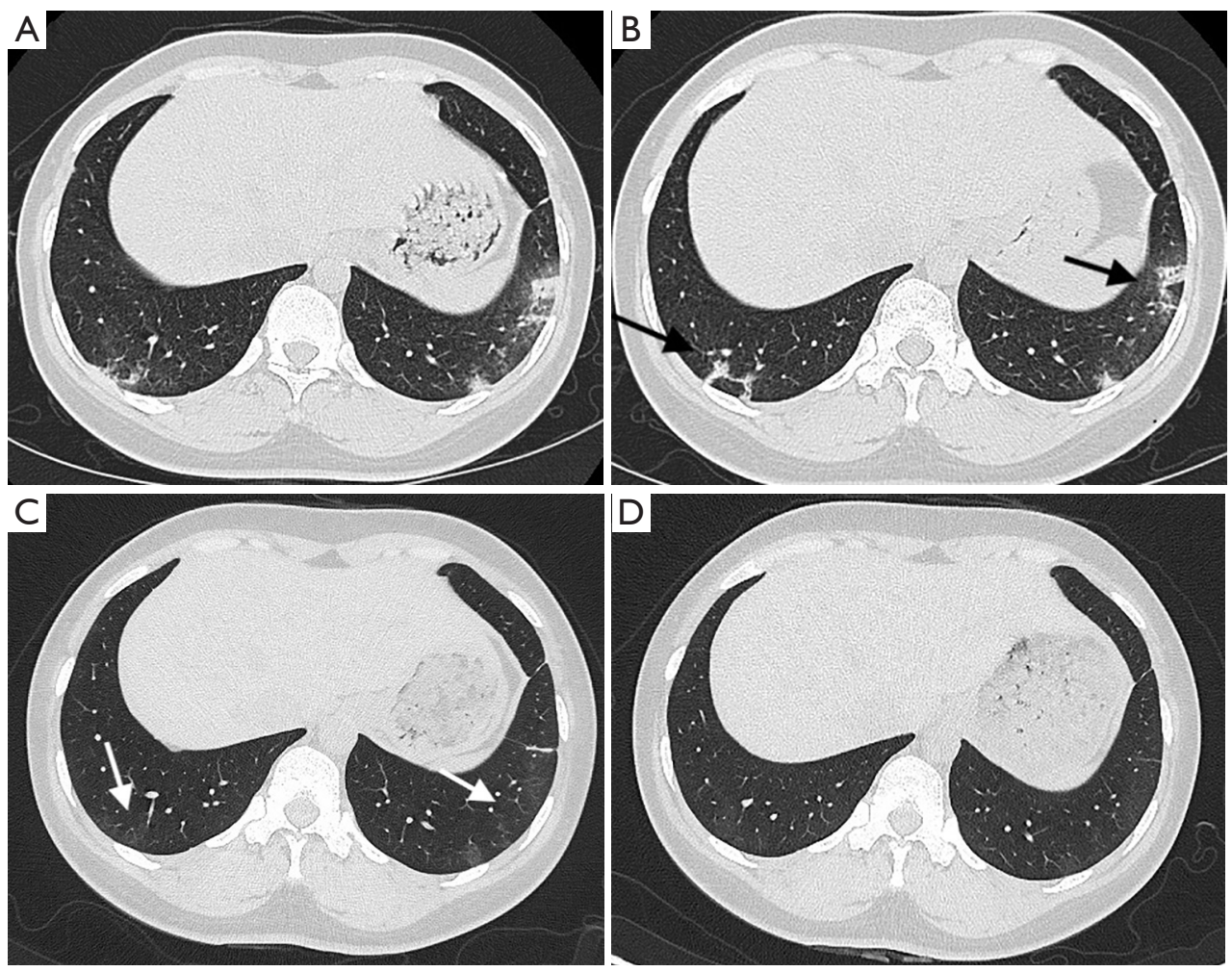

Figure 1 Chest CT images of a 36-year old woman with COVID-19. (A) Her onset CT images, with GGO and reticulation in the peripheral and subpleural area. (B) Absorption of the GGO and progressive reticulation (black arrow) as seen on January 31, 2020, when her RT-PCR result was negative (twice consecutively, after at least a 24-hour interval). (C) The previous lesions changed to GGO (white arrow) on February 22, 2020, when the RT-PCR retested positive. (D) Improvements on chest CT 10 days after the second hospitalization. GGO, ground glass opacity.

results. Excellent specimen types and the best timing for peak viral load of this disease still need to be further determined (10). A study on the characteristics of the nucleic acid conversion from 70 patients with COVID-19 found that 15 (21.4\%) patients experienced positive RTPCR retest results for SARS-CoV-2 after two consecutive negative results, and most of them had relief in symptoms or radiographic features (11). They suggested that this could be related to the false-negative results seen in RTPCR tests, and these patients had a longer viral clearance period, instead of them "turning positive again" or "reoccurring." Yuan et al. (12) reported their observation of 25 COVID-19 patients who had retested positive in their RT-PCR tests after discharge; they found that the CT scan results of 20 patients showed improvement or did not worsen compared to their first discharge CT scan. In our study, GGOs and reticulations were observed in most of the patients, and the lesions decreased in size and the number of regions affected on follow-up CT scans, which was in concordance with the study by Yuan et al. (12). However, six patients showed changes when their RT-PCR retested positive after discharge, there was a transformation from reticulation to GGO, or a re-appearance or increase of GGOs in the previous regions. This sign may indicate that the virus can hide in the body, and the patients were not actually completely recovered. As we know, the absorption of GGOs and the appearance of more reticulation are normally considered as part of the repairing process, so these changes seen on the chest CT scans could be very helpful when we observe and monitor the patients with negative RT-PCR. This is because it could remind us that the virus could still be present in the body, and that there is a good chance of obtaining a positive PCR test again. Two patients in our study did not show any lesions in either of their lungs when their PCR retested positive, which further implied the inconsistency between CT and PCR results. 

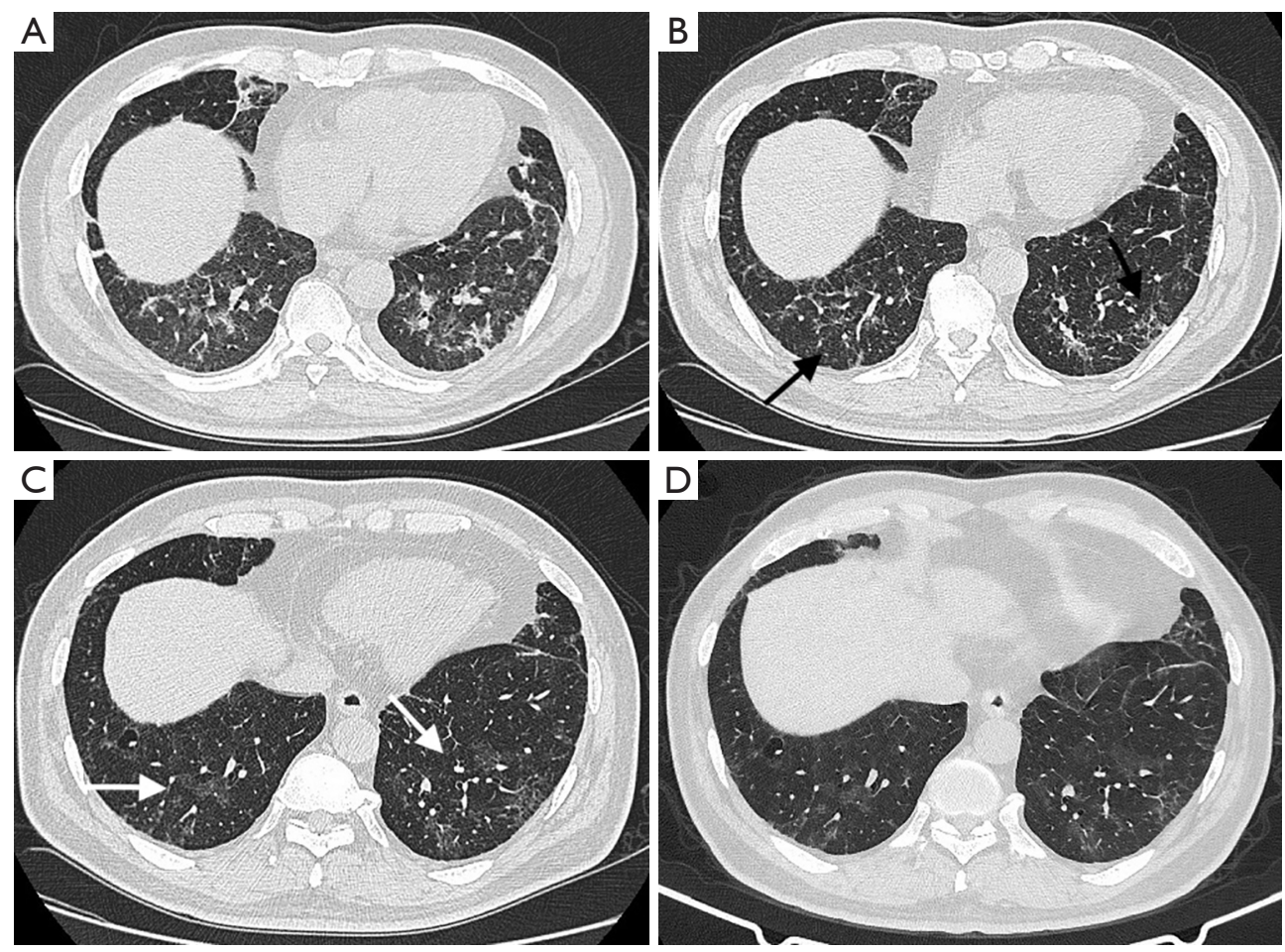

Figure 2 Chest CT images of a 57-year old man with COVID-19. (A) His onset CT images, with multiple GGOs and reticulations on February 4, 2020. (B) The absorption of the GGOs (black arrow) on February 21, 2020, when his RT-PCR result was confirmed to be negative (twice consecutively, after an interval of at least 24 hours). (C) The previous lesions changed to GGOs (white arrow) on March 9 , 2020, when the RT-PCR test retested positive. (D) A GGO with lower density 8 days after re-admission. GGO, ground glass opacity.

A cohort study of 1014 patients mentioned that more than $70 \%$ of suspicious patients with negative RT-PCR tests had typical CT manifestations (13), but on the other hand, there were asymptomatic cases with positive RT-PCR results (14). Therefore, the combination of both CT and PCR is still vital in future follow-up and medical surveillance.

SARS-CoV-2 is insidious, and it could lurk in human bodies longer than we thought. Patients who meet the current standards of discharge could still carry the virus. We are not sure of the relationship between the traces of virus detected and its transmission abilities at the moment, but for the safety of the public, longer observation times must be considered for these patients. Because most patients do not have worsening conditions when re-admitted, it is hard to determine the specific clinical characteristics that could predict the recurrence of the virus. Two negative RTPCR tests at 24-hour apart may not be sufficient for viral clearance evaluation, and current discharge standards may need to be reevaluated or adjusted (15). In our opinion, quarantining at home after discharge is still necessary, and a bi-monthly follow-up is recommended. The specific CT manifestation that we observed, that is, the transformation from reticulation to GGO, might be a sign of the existence of the virus and the patient is not fully recovered; therefore, we should give great attention for these circumstances. An inconsistency could exist between CT and PCR, but they also supplement each other, which is why we need to combine both of them appropriately during early diagnosis and follow-up.

\section{Acknowledgments}

We wish to express our sincere thanks to Yujie Yuan for his professional help and constructive suggestions for this article.

Funding: None. 


\section{Footnote}

Conflicts of Interest: All authors have completed the ICMJE uniform disclosure form (available at http://dx.doi. org/10.21037/qims-20-530). The authors have no conflicts of interest to declare.

Ethical Statement: This study was approved by the institutional review board of the Public Health and Clinical Center of Chengdu. The requirement for informed consent was waived.

Open Access Statement: This is an Open Access article distributed in accordance with the Creative Commons Attribution-NonCommercial-NoDerivs 4.0 International License (CC BY-NC-ND 4.0), which permits the noncommercial replication and distribution of the article with the strict proviso that no changes or edits are made and the original work is properly cited (including links to both the formal publication through the relevant DOI and the license). See: https://creativecommons.org/licenses/by-nc-nd/4.0/.

\section{References}

1. National Health Commission of the People's Republic of China. Update on the epidemic situation of the novel coronavirus pneumonia as at 24:00 on 4 Mar 2020. Beijing, China: National Health Commission of the People's Republic of China, 2020.

2. National Health Commission of the People's Republic of China. Diagnosis and treatment protocols of pneumonia caused by a novel coronavirus. Beijing, China: National Health Commission of the People's Republic of China, 2020.

3. Li Y, Yao L, Li J, Chen L, Song Y, Cai Z, Yang C. Stability Issues of RT-PCR Testing of SARS-CoV-2 for Hospitalized Patients Clinically Diagnosed with COVID-19. J Med Virol 2020. doi: 10.1002/jmv.25786.

4. Yin Y, Wunderink RG. MERS, SARS and other coronaviruses as causes of pneumonia. Respirology 2018;23:130-7.

5. Huang C, Wang Y, Li X, Ren L, Zhao J, Hu Y, Zhang L, Fan G, Xu J, Gu X, Cheng Z, Yu T, Xia J, Wei Y, Wu W, Xie X, Yin W, Li H, Liu M, Xiao Y, Gao H, Guo L, Xie J, Wang G, Jiang R, Gao Z, Jin Q, Wang J, Cao B. Clinical features of patients infected with 2019 novel coronavirus in Wuhan, China. Lancet 2020;395:497-506.

6. Ye G, Pan Z, Pan Y, Deng Q, Chen L, Li J, Li Y, Wang $X$. Clinical characteristics of severe acute respiratory syndrome coronavirus 2 reactivation. J Infect 2020;80:e147.

7. Xing Y, Mo P, Xiao Y, Zhao O, Zhang Y, Wang F. Postdischarge surveillance and positive virus detection in two medical staff recovered from coronavirus disease 2019 (COVID-19), China, January to February 2020. Euro Surveill 2020;25:2000191.

8. Xie X, Zhong Z, Zhao W, Zheng C, Wang F, Liu J. Chest CT for typical 2019-nCoV pneumonia: relationship to negative RT-PCR testing. Radiology 2020. doi: 10.1148/ radiol.2020200343.

9. Chen D, Xu W, Lei Z, Huang Z, Liu J, Gao Z, Peng L. Recurrence of positive SARS-CoV-2 RNA in COVID-19: A case report. Int J Infect Dis 2020;93:297-9.

10. Tahamtan A, Ardebili A. Real-time RT-PCR in COVID-19 detection: issues affecting the results. Expert Rev Mol Diagn 2020;20:453-4.

11. Xiao AT, Tong X, Zhang S. False-negative of RT-PCR and prolonged nucleic acid conversion in COVID-19: Rather than recurrence. J Med Virol 2020. doi: 10.1002/ jmv.25855.

12. Yuan J, Kou S, Liang Y, Zeng J, Pan Y, Liu L. PCR Assays Turned Positive in 25 Discharged COVID-19 Patients. Clin Infect Dis 2020. doi:10.1093/cid/ciaa398.

13. Ai T, Yang Z, Hou H, Zhan C, Chen C, Lv W, Tao Q, Sun Z, Xia L. Correlation of chest CT and RT-PCR testing in coronavirus disease 2019 (COVID-19) in China: a report of 1014 cases. Radiology 2020. doi:10.1148/ radiol.2020200642.

14. Xu R, Du M, Li L, Zhen Z, Wang H, Hu X. CT imaging of one extended family cluster of corona virus disease 2019 (COVID-19) including adolescent patients and "silent infection". Quant Imaging Med Surg 2020;10:800-4.

15. Lan L, Xu D, Ye G, Xia C, Wang S, Li Y, Xu H. Positive RT-PCR test results in patients recovered from COVID-19. JAMA 2020. doi:10.1001/jama.2020.2783.

Cite this article as: Li C, Luo F, Xie L, Gao Y, Zhang N, Wu B. Chest CT study of fifteen COVID-19 patients with positive RT-PCR retest results after discharge. Quant Imaging Med Surg 2020;10(6):1318-1324. doi: 10.21037/qims-20-530 